Service social

\title{
Le travailleur social et les situations d'urgence
}

\section{May Hazzaz}

Volume 34, numéro 1, 1985

Personnes âgées, milieux de vie et pratiques sociales

URI : https://id.erudit.org/iderudit/706256ar

DOI : https://doi.org/10.7202/706256ar

Aller au sommaire du numéro

Éditeur(s)

École de service social de l'Université Laval

ISSN

1708-1734 (numérique)

Découvrir la revue

Citer cet article

Hazzaz, M. (1985). Le travailleur social et les situations d'urgence. Service social, 34(1), 148-157. https://doi.org/10.7202/706256ar d'utilisation que vous pouvez consulter en ligne.

https://apropos.erudit.org/fr/usagers/politique-dutilisation/ 

formation sociale de l'Université SaintJoseph de Beyrouth.

\section{Le travailleur social et les situations d'urgence}

\section{May Hazzaz}

Pour la Corporation professionnelle des travailleurs sociaux du Québec, l'objectif majeur de la profession est centré sur "l'être social", c'est-à-dire la personne humaine en constante relation avec son environnement. Le travailleur social a, par ailleurs, au cours de sa formation, acquis et expérimenté différentes méthodes qui lui permettent aujourd'hui de comprendre les besoins d'une personne, d'un groupe et d'une communauté.

En prenant comme exemple une journée des dernières années, regardons comment évolue notre environnement.

Pour la seule journée du 29 novembre 1983, mes étudiantes et moi avions retenu une dizaine d'incidents majeurs en lisant le quotidien libanais de langue française L'Orient-le Jour, et ce en excluant les événements du Liban et du Moyen-Orient :

- Écrasement d'un Boeing 707 à Madrid : 186 morts, 11 survivants.

- Tempête à Denver aux États-Unis: 48 personnes tuées et plusieurs blessées.

- Affrontements sanglants entre forces de l'ordre et manifestants au Bengladesh : 5 morts, plusieurs centaines de blessés ; couvrefeu imposé à Dacca, la capitale.

— Un avion nigérien s'écrase à 600 kilomètres de Lagos : 53 morts, des dizaines de disparus.

- Guerre nucléaire : l'Europe serait menacée de disparition. Privées d'abris antiatomiques, des dizaines de milliers de personnes succomberaient aux radiations.

- Selon le Jérusalem-Post d'Israël, aujourd'hui, 29 novembre 1983, serait le début de "la fin du monde". 
Plus près de nous, le journal québécois Le Soleil du 4 septembre 1984 rapportait divers incidents, les uns mineurs et d'autres majeurs :

- Une bombe fait 3 morts et 40 blessés à la Gare centrale de Montréal.

- Une tornade en Ontario cause pour quelques millions de dollars de dommages à plus de six cents maisons et commerces en plus de blesser une trentaine de personnes.

- Sur la route de Rivière-du-Loup, un autobus scolaire dérape et se renverse dans un fossé : un mort et 14 blessés parmi les adolescents.

- À Sainte-Foy, plusieurs incendies suspects se déclarent.

- Dans le Nord québécois, catastrophe écologique : vingt milles caribous se noient à cause de la crue exceptionnelle d'une rivière.

- À Sydney, Australie, une querelle entre motards Bandidos et Commencheros fait sept victimes.

- À Johannesburg, Afrique du Sud, au moins 14 personnes ont été tuées et 31 blessées, au cours des émeutes qui ont secoué six banlieues noires, au sud de la capitale.

- En Corée du sud, 2000 victimes suite à des inondations à Séoul : 86 personnes sont décédées, 40 portées disparues et 98 blessées ; les dégâts sont estimés à 17 millions de dollars.

- À Manille, aux Philippines, le cyclone Ike a fait plus de 332 morts, 91 disparus, des centaines de blessés et a laissé plus d'un million de personnes sans abri, selon une estimation faite la nuit dernière par le Ministère philippin des services sociaux.

Cette brève liste d'événements nous donne une idée de l'environnement où le monde dans lequel nous vivons évolue, et laisse aussi percevoir comment le développement de la notion de risque caractérise notre époque.

Les sinistres naturels ont toujours existé ; indissociables de l'univers, ils font partie de la grande histoire de l'humanité. Même si l'ampleur du risque nous dépasse, faut-il rester les bras croisés?

Pour certains professionnels de la protection civile, ${ }^{1}$ la transformation du mode de vie, la conquête de nouvelles sources d'énergie, la maîtrise progressive de la matière, l'existence d'une civilisation de loisirs ont, pour contrepartie, la permanence du risque.

Par ailleurs, la réaction de dépendance et l'interaction des uns par rapport aux autres rendent l'analyse des risques encore plus complexe et difficile. Peut-on comparer l'incendie d'une échoppe d'artisan du $17^{\mathrm{e}}$ siècle à celui qui éclate dans un édifice résidentiel d'une ville à forte densité, dans une usine, ou dans une centrale nucléaire? Plus les 
sociétés actuelles aspirent à un mieux-vivre, plus elles deviennent vulnérables. Ampleur, permanence, complexité des risques; avec, en face, les risques mineurs mais quotidiens.

Les catastrophes naturelles nous font subir des pertes énormes : en contrepartie, c'est plus de vingt mille à trente-cinq mille morts que causent chaque année les accidents de tout ordre, les risques quotidiens de la route, du travail, dans les foyers, dans la nature, dans les loisirs. On a raison de croire que trop de blessures et de morts résultent de l'inconscience, de l'ignorance; trop de forêts brûlent par suite d'imprudence et d'indifférence. Ce sont des risques qui sont l'ouvre de l'homme contre lui-même ou contre les autres hommes. Cet état de chose, et c'est normal, provoque un besoin de sécurité et certains vont même jusqu'à parler de droit à la sécurité. Certaines sociétés modernes sont même marquées

"par un transfert de plus en plus poussé du risque et de ses conséquences de l'individu vers la collectivité. Cette évolution aboutit trop souvent à une régression de la responsabilité personnelle qui conduit à assigner à la cité [l'État] un rôle protecteur sans cesse croissant. Il faut savoir aussi que le risque comporte une part d'inéluctable qu'aucune organisation sociale ne pourra jamais réduire, il est par conséquent intimement lié au comportement des individus qui ont trop souvent tendance à se reposer sur l'intervention de la puissance publique.$^{2}$

À mon avis, il ne sert à rien que l'État renforce ou améliore, d'année en année, la quantité ou la qualité de ses moyens de secours et de soins s'il n'a pas en face de lui des interlocuteurs valables.

Dans la lutte contre tous les genres de risques, il est nécessaire que chacun se sente concerné et responsable. II ne peut y avoir de véritable sécurité que si les citoyens sont conscients de leurs responsabilités. Pour cela, il faut qu'ils soient informés. Mais, parallèlement, ne faudrait-il pas que nous soyons nous-même, en tant que travailleur social, formé et informé?! Comment identifier une situation d'urgence? Comment classifier ces sinistres, naturels ou faits par la main de I'homme, volontairement ou inconsciemment? Que pensez-vous que nous pouvons faire? Comment faut-il le faire? et pourquoi? Quelles peuvent être les diverses caractéristiques de l'intervention en situation d'urgence?

Sous le mot urgence, j'inclus plusieurs notions dont, bien sûr, en premier lieu, la guerre avec ses conséquences désastreuses comme la violence, le terrorisme, la perte d'êtres chers et de biens, la destruction, le déplacement des populations, les blessures physiques et mentales, les privations matérielles et psychologiques. 
Deuxièmement, il y a la notion de sinistre naturel, mineur ou majeur, tellurique, climatique ou technologique. En voici des exemples : les tremblements de terre, les glissements et effondrements de terrain, une tempête, un cyclone, une inondation, une panne d'électricité, un incendie, le maniement de produits chimiques ou radioactifs dangereux, etc.

Troisièmement, la notion de crises situationnelles spécifiques englobe le problème de licenciements en groupe, la drogue, le deuil, I'hospitalisation, la suite d'une épidémie, le changement de statut/rôle.

Et, en quatrième lieu, il faut penser aux crises de maturation qui sont propres aux étapes de la vie (enfance, adolescence, âge adulte, ménopause, vieillesse).

Pourquoi la notion de crise ? Le mot grec krisis signifie "décision" et ce mot porte ici une connotation de moment décisif, de point tournant dans la vie d'une personne, d'une famille, d'un groupe, d'une collectivité ou communauté, d'un pays. En médecine, il désigne souvent dans le cours d'une maladie le changement vers, soit la guérison soit la mort. Dans les relations internationales, il désigne aussi un événement ou un conflit dont l'issue détermine la paix ou la guerre.

Parallèlement à cette première classification, le Ministère de la santé et du bien-être social (1977) a, pour sa part, condensé, dans un schéma très intéressant, les trois sortes de catastrophes susceptibles d'arriver au Canada : naturelles, industrielles et sociales ou politiques. ${ }^{3}$ (Voir Annexe.)

Tout ceci, encore, peut nous faire prendre conscience de la menace constante de catastrophes, mais toutes personnes ou communautés, tous groupes ou pays vivent dans l'espoir qu'ils ne seront jamais touchés eux-mêmes. La vérité est que les sinistres peuvent arriver à n'importe qui, n'importe quand.

Mais comment peut-on définir le sinistre? Allen Barton donne cette définition qui me semble la plus globale et la plus adéquate :

"Un sinistre est une situation de stress collectif, dans laquelle une collectivité n'arrive plus à avoir les conditions nécessaires de survie telles la sécurité, la nourriture, un abri, un revenu, une information, pour poursuivre ses activités quotidiennes. ${ }^{4}$

D'ailleurs, plusieurs auteurs se sont penchés sur l'analyse de cette notion de sinistre, chacun mettant l'accent sur l'un ou l'autre des divers processus en cours.

Howard Parad ${ }^{5}$ va encore plus loin et précise que ce "stress collectif» peut surgir à partir d'éléments externes ou internes au système en place. Les sinistres provenant de sources externes au système et provoquant de grands changements dans l'environnement 
physique sont : les tremblements de terre, les inondations, les glissements de terrain, les tempêtes, les explosions industrielles ou chimiques, etc., et ceux provenant de sources internes au système : les grèves, les émeutes, les attentats, les coups d'État, les guerres, civiles ou non, provoquant ainsi un "changement surtout au plan socioéconomico-politique.

Ainsi la guerre au Liban et les bombardements fréquents, anarchiques, imprévisibles et non maîtrisables de quartiers résidentiels, a provoqué et continue à provoquer, non seulement un changement d'ordre physique (villages détruits, quartiers urbains fantômes, séparation de la capitale en Est-Ouest), mais surtout un changement aux niveaux des mentalités (systèmes de valeurs, idéologie, philosophie), du système économique (nouvelle forme d'investissement, immobilisme du secteur industriel, blocage monétaire, et bancaire) et du système politique et administratif (décentralisation, régionalisation, implantation, confédération, etc.).

La différence entre des sinistres naturels comme une inondation ou un tremblement de terre, c'est que le premier peut être prévisible et la situation postérieure plus ou moins contrôlable, alors que le second a un caractère soudain, imprévisible, incontrôlable, suscitant ou nécessitant parfois des changements plus importants au niveau physique et géologique.

Reuben Hill ${ }^{6}$ soutient que, indépendamment de la nature interne ou externe du sinistre, ce dernier ne découle pas d'un seul phénomène; il faut le concours de plusieurs facteurs pour qu'un incident atteigne des propositions désastreuses. Les recherches révèlent qu'environ soixante situations diverses peuvent entraîner un désastre comme, par exemple: l'écroulement d'un édifice comme un centre commercial ; un accident de chemin de fer sur une voie publique ; l'écrasement d'un avion de passagers sur un village ou dans une ville; l'explosion d'un camion transportant des produits chimiques ou explosifs.

Quelles sont donc par conséquent les variables dont il faut tenir compte pour qu'un incident de ce genre entraîne une situation d'urgence? Indépendamment de son origine interne ou externe, les caractéristiques propres à tout sinistre sont, particulièrement: sa soudaineté, son étendue, les possibilités de sa prévision, de son contrôle ou de sa maitrise; l'ampleur de ses effets ou conséquences désastreuses; les ressources disponibles et accessibles: internes, externes, humaines et matérielles; la perception objective et subjective que la population se fait du sinistre en tant qu'événement.

Quant à l'étendue et à la durée d'un sinistre, elles peuvent, dans certains cas, se limiter dans le temps et l'espace, avoir lieu dans une 
ville ou une région bien déterminée, se dérouler dans un temps allant de quelques minutes à quelques heures. Par contre, dans le cas du Liban, le sinistre a bien commencé dans un quartier urbain, populeux, mais il a fini par s'étendre d'une ville à un village, d'une région du Sud à la plaine de la Bekaa, puis au Mont-Liban et au Nord, pour finalement englober le pays en entier; il a commencé au matin du dimanche 13 avril 1975, a duré plusieurs années et dure encore.

Pour ce qui est des phases, Powell et Rayner ${ }^{7}$ pensent que le processus peut être divisé en sept phases : menace - alerte - impact - inventaire - secours - orientation - récupération. Par ailleurs, Max Siporin ${ }^{8}$ voudrait circonscrire les sept phases en trois étapes effectives : phase de l'alerte; phase de confusion et de désorganisation; et phase de réorganisation et réhabilitation des personnes, des familles, et de la vie communautaire.

Personnellement, je pense qu'on peut effectivement retenir trois phases essentielles :

- au moment même du sinistre: phase de panique, confusion, désorganisation ;

- phase de réajustement à court terme: évacuation, accueil, hébergement, alimentation, information, aide financière ;

- phase de réorganisation à long terme : reconstruction, relocalisation, restauration physique et psychologique.

À mon avis, cette subdivision nous aide à formuler une meilleure et rapide analyse de la situation, à porter un premier diagnostic, et ainsi à passer plus rapidement à l'action.

Parlant de prévention dans le domaine des soins de santé mentale, Van Antwerp ${ }^{9}$ constatait que l'on ne pouvait plus traiter les malades comme dans le passé : il faudrait intervenir tant auprès des individus que dans les systèmes sociaux avant que les troubles ne se manifestent. Dans les situations d'urgence, le mot "intervenir» prend toute son importance.

Si en latin intervenire veut dire : prendre part volontairement, le Larousse, lui, ajoute une autre dimension : intervenir c'est "venir avec", "venir entre». Ce qui suppose, à mon avis, une connaissance préalable de la situation pour pouvoir, librement et volontairement, agir pendant et planifier pour après, tant auprès de l'individu que dans les systèmes sociaux.

Par conséquent, intervenir dans les situations d'urgence exige une double action de prévention et de coordination des secours, indispensables l'une et l'autre pour comprendre aujourd'hui les besoins d'une communauté et assurer une protection efficace à la population. 
Jusqu'à il n'y a pas longtemps, on présentait le service social comme un art et une science, ou encore comme une profession d'aide où les relations interpersonnelles étaient privilégiées; personnellement, je voudrais juste rappeler que c'est aussi une profession d'action. Être travailleur social, c'est accepter de vivre une aventure et, dans toute aventure, il y a toujours des risques; on s'engage pour le meilleur et le pire. Être travailleur social, c'est passer autant de temps "dans la rue» que dans l'intimité d'un bureau, c'est savoir sortir de son organisme parce que les urgences ont lieu le plus souvent - pour ne pas dire toujours - dans la rue, dans les maisons, dans les lieux de travail, dans les quartiers. En d'autres termes, savoir se remettre dans la peau d'un travailleur social extra muros ou detached worker, comme le service social l'a connu à ses débuts. Car, quand nous sommes dans la rue, pour ainsi dire, nous vivons la réalité quotidienne de notre clientèle, et ainsi nous pouvons prendre conscience de l'évolution et de l'urgence de certaines situations : on ne peut plus attendre que les gens viennent à nous réclamer aide ou droits; il faut aller vers eux.

L'aide à apporter doit être immédiate, rapide, de courte ou moyenne durée et d'accès facile. L'aide est bien sûr apportée à tous, sans discrimination aucune - ni ethnique, ni religieuse, ni de classe, ni de couleur, ni de nationalité - mais la priorité est évidemment accordée à ceux qui ont le moins de chance de s'en sortir seuls.

L'entrevue est ici un instrument, non pour connaître l'histoire ou le passé de la personne ou de la famille sinistrée, mais son présent le "ici et maintenant" y prend toute son importance - comme dans l'approche à court terme.

Le temps, le nombre, l'ampleur et la complexité sont autant de facteurs qui nous obligent à comprendre les besoins de la communauté et à composer avec la situation réelle.

L'action à entreprendre est pensée et planifiée pour l'immédiat avec la possibilité d'un court, moyen ou long terme, suivant le cas chacun utilisant ses aptitudes et habiletés personnelles, recherchant les moyens que la situation lui impose, si minimes soient-ils.

Par ailleurs, comme le mentionnent Aguilera et Messick, 10 il faut démystifier l'opinion selon laquelle seuls les professionnels possédant une formation supérieure ou universitaire peuvent intervenir efficacement. Nous aurons à collaborer avec des personnes ayant peu ou pas de formation formelle. Pour ma part, j'ai eu l'occasion de travailler avec des groupes volontaires de jeunes et de moins jeunes, des spécialistes et des analphabètes; ces derniers ont souvent fait preuve d'une compréhension et d'une efficacité égales sinon supérieures à 
celles des experts ou spécialistes dans l'approche des personnes en difficulté ou de situations de stress collectif.

Travailler avec, ou faire travailler des volontaires, c'est encore bien autre chose que de se retrouver "parachuté" au sein d'une équipe pluridisciplinaire avec d'autres professionnels. Comment répartir les rôles? Qui doit faire quoi?

II ne faut pas croire, non plus, que toute population en situation de stress ou sinistrée est nécessairement "hébétée, passive et apathique" ; très souvent elle est la première à se prendre en main. II faut, par conséquent, savoir utiliser ses ressources internes et surtout humaines. Ce sont là, à mon avis, les caractéristiques, contraintes et limites de l'intervention sociale en situation d'urgence. Face à ces nouveaux problèmes, nous avons le devoir de penser à de nouveaux rôles ou alternatives, faute de trouver des solutions rapides ou radicales.

$\mathrm{Si}$ nous ne pouvons pas éliminer ou enrayer certains sinistres naturels ou créés par la main de l'homme contre l'homme... au moins pouvons-nous travailler à minimiser leurs effets et leurs conséquences désastreuses. 


\section{Références bibliographiques}

1 C. Gérondeau, C. Bonnet, H. Tazieff et P. Pisa, cités dans : Revue d'information de la sécurité civile, no 1, 1979.

2 C. Bonnet, Revue d'information de la sécurité civile, no 1, 1979.

3 Ministère de la santé et du bien-être social, Planification d'urgence pour les installations de soins spéciaux, Ottawa, 1977.

4 A. Barton, Communities in Disaster, New York, Doubleday, 1969, p. 7.

${ }^{5}$ H. PARAD, Emergency and Disaster Management, New York, Bowie Charles Press, 1976.

6 R. HILL, "Generic features of families under stress», dans: H. PARAD (éd.), Crisis Intervention : Selected Readings, New York, Family Service Association of America, 1965 : 32-52.

7 A. Powell et B. Raynor, Progress Notes : Disaster Investigations, Washington, Chemical Corps Medical Laboratories, 1952.

${ }^{8}$ M. SIPORIN, "Disaster aid", Encyclopedia of Social Work, vol. 16, 1978 : 245-254.

9 M. VAN ANTWERP, "The route to primary prevention", Community Mental Health Journal, vol. 7, no 3, 1971 : 183-188.

10 D. Aguilera et J. Messick, Intervention en situation de crise, Toronto, Mosby, 1976. 


\section{ANNEXE}

\section{Identification des types de catastrophes susceptibles d'arriver au Canada}

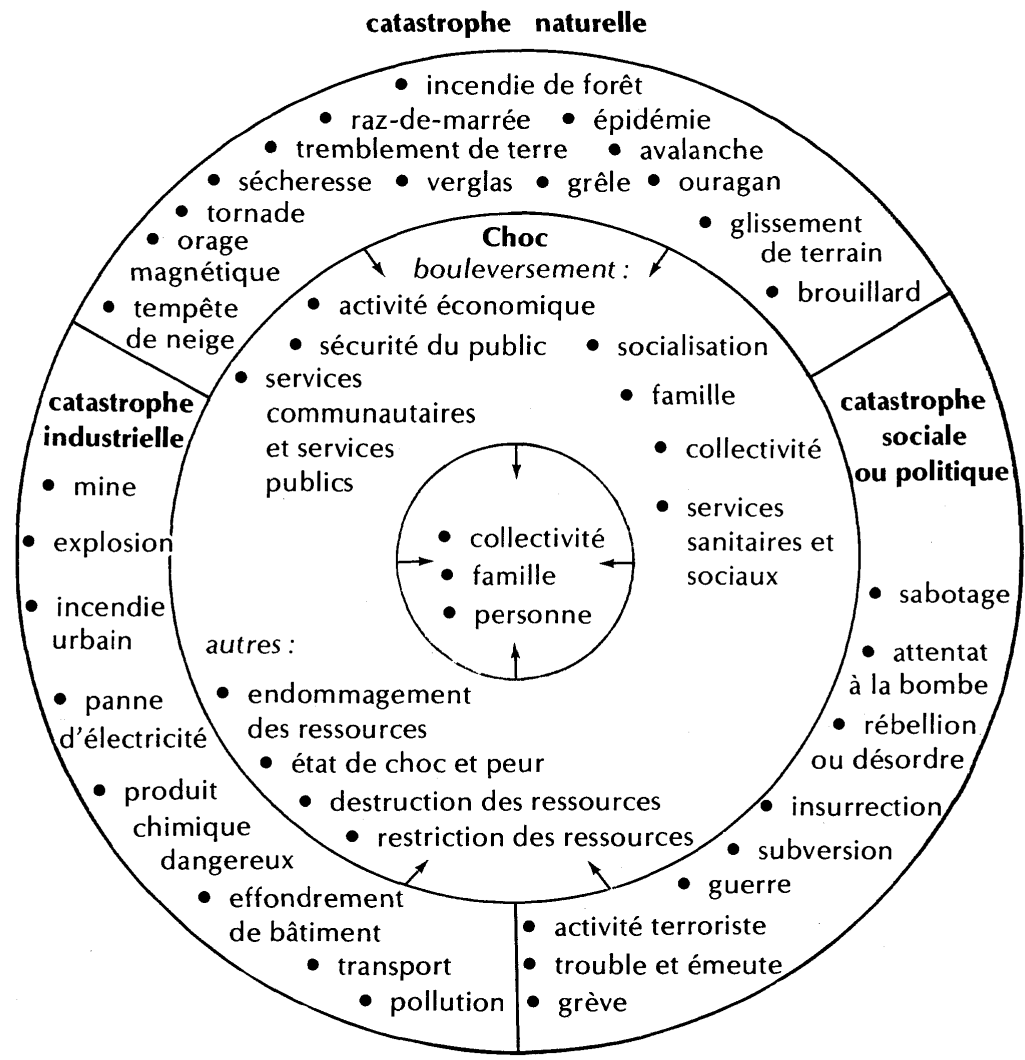

Source: Ministère de la santé et du bien-être social, Planification d'urgence pour les installations de soins spéciaux, Ottawa, 1977. 